\title{
Panax Notoginseng Protects against Diabetes-Associated Endothelial Dysfunction: Comparison between Ethanolic Extract and Total Saponin
}

\author{
Xutao Zhang, ${ }^{1}$ Chunxiu Zhou, ${ }^{1}$ Lingchao Miao, ${ }^{1}$ Yi Tan, ${ }^{1}$ Yan Zhou, ${ }^{1}$ Meng Sam Cheong, \\ Yu Huang, ${ }^{2}$ Yitao Wang ${ }^{1},{ }^{1}$ Hua Yu, ${ }^{1}$ and Wai San Cheang ${ }^{1}{ }^{1}$ \\ ${ }^{1}$ State Key Laboratory of Quality Research in Chinese Medicine, Institute of Chinese Medical Sciences, University of Macau, \\ Macao SAR, China \\ ${ }^{2}$ School of Biomedical Sciences, Chinese University of Hong Kong, Hong Kong SAR, China \\ Correspondence should be addressed to Wai San Cheang; annacheang@um.edu.mo
}

Xutao Zhang and Chunxiu Zhou contributed equally to this work.

Received 24 June 2021; Accepted 7 August 2021; Published 6 September 2021

Academic Editor: Yanwei Xing

Copyright (c) 2021 Xutao Zhang et al. This is an open access article distributed under the Creative Commons Attribution License, which permits unrestricted use, distribution, and reproduction in any medium, provided the original work is properly cited.

\begin{abstract}
Previous studies revealed a cardioprotective potential of Panax notoginseng to relieve acute myocardial infarction and focal cerebral ischemia-reperfusion. However, whether $P$. notoginseng protects endothelial function in diabetes and the underlying mechanisms remain to be explored. P. notoginseng contains several chemical components including saponins, which are commonly believed as the major bioactive ingredients. The present study was aimed to examine and compare the vasoprotective effects of the ethanolic extract of $P$. notoginseng (PNE) and total saponin (PNS). Both aortas and carotid arteries were isolated from male C57BL/6J mice for ex vivo treatment with risk factors (high glucose or tunicamycin) with and without the presence of PNS and PNE. Diabetic model was established by feeding the mice with a high-fat diet ( $45 \%$ kcal\% fat) for 12 weeks, while PNS and PNE were administrated by oral gavage at $20 \mathrm{mg} / \mathrm{kg} /$ day for another 4 weeks. Ex vivo exposure to high glucose impaired acetylcholine-induced endothelium-dependent relaxations in mouse aortas, decreased phosphorylation of AMPK and eNOS, and induced endoplasmic reticulum (ER) stress and oxidative stress. These effects were reversed by cotreatment of PNS and PNE with PNS being more potent. Furthermore, the vaso-protective effects were abolished by Compound C (AMPK inhibitor). Chronic treatment with PNS and PNE improved endothelium-dependent relaxations and alleviated ER stress and oxidative stress in aortas from high-fat diet-induced obese mice. PNE was more effective to improve glucose sensitivity and normalize blood pressure in diabetic mice. The present results showed that PNS and PNE reduced ER stress and oxidative stress and, subsequently, improved endothelial function in diabetes through AMPK activation. This study provides new inspiration on the therapeutic potential of $P$. notoginseng extract against vascular diseases associated with metabolic disorders.
\end{abstract}

\section{Introduction}

Panax notoginseng (Burk) F.H. Chen is a widely used medicinal plant in East Asian countries for thousands of years. Radix Notoginseng, also named as Sanqi or Tianqi in Chinese, is the dried root of $P$. notoginseng, which is the main part used for medical purpose, while the leaves, fruits, and flowers possess medicinal value [1]. According to the Chinese Pharmacopoeia, its traditional pharmacological effect is to disperse blood stasis and stop bleeding, reduce swelling and relieve pain. Increasing pharmacological and clinical evidence have supported that $P$. notoginseng, particularly its saponin components, possess multiple beneficial effects. $P$. notoginseng saponins (PNS) and ginsenosides $\mathrm{Rb} 2$ and Rd2 inhibit platelet activity [2-4]. PNS exhibit antiobesity [5] and antidiabetic properties [6] and also attenuate nonalcoholic fatty liver disease [7]. Of note, $P$. notoginseng is found to be cardio-protective as PNS protect against acute 
myocardial infarction in mice [8] and relieve the focal cerebral ischemia-reperfusion in rats [9], while notoginsenoside $\mathrm{R} 1$ promotes angiogenic activity in endothelial cells and zebrafish [10]. Furthermore, notoginsenoside R1 was reported to prevent cardiomyocyte apoptosis and ischemic/reperfusion injuries via inhibition of signaling pathways related to oxidative stress and endoplasmic reticulum (ER) stress [11]. Another recent study showed that PNS protect against thapsigargin-induced mitochondrial injury, reducing ROS accumulation and $\mathrm{Ca}^{2+}$-dependent ER stress [12].

ER is an important organelle for the synthesis and folding of membrane and secretory proteins, but disruption of ER function leading to ER stress is a critical contributor to cellular dysfunction and metabolic disorder and related complications [13-15]. Complex signaling cascades are activated in response to ER stress, initiated by phosphorylation of three ER membrane-associated proteins: PKR-like endoplasmic reticulum kinase (PERK), inositol requiring enzyme 1 (IRE1), and activating transcription factor 6 (ATF6) [16]. A crosstalk is suggested between ER stress and oxidative stress in cardiovascular diseases [17]. The production of reactive oxygen species (ROS) is elevated during ER stress via NADPH oxidases, mainly Nox2 and Nox4 [18]. On the other hand, ROS accumulation further promotes ER stress [19], where mitochondrial ROS plays an important role [20]. In consistence, our previous findings support that ER stress induces ROS production and endothelial dysfunction; and that alleviation of ER stress and oxidative stress protects against diabetic vasculopathy $[21,22]$.

$5^{\prime}$-Adenosine monophosphate-activated protein kinase (AMPK) plays a key role both in regulating metabolic homeostasis and cardiovascular biology; its phosphorylation contributes to the activation of endothelial nitric oxide synthase (eNOS) [23]. AMPK activation was shown to mitigate ER stress and oxidative and rescue vascular function in diabetes $[24,25]$.

Previous studies showed that $P$. notoginseng, in particular PNS, improves vascular and metabolic functions. However, little is known whether $P$. notoginseng protects endothelial function in diabetes and the underlying mechanisms. Importantly, most studies showed PNS as the major bioactive components of $P$. notoginseng. Herein, we examined and compared the vaso-protective effects of total ethanolic extract of $P$. notoginseng (PNE) and PNS in alleviating ER stress and endothelial dysfunction associated with diabetes in vitro and in vivo.

\section{Materials and Methods}

2.1. Preparation of Herbal Extract. The air-dried and powered Panax notoginseng powder (Sanqi or Tianqi) was extracted with $95 \%$ ethanol $(1: 10 \mathrm{w} / v)$ and refluxed for $1 \mathrm{~h}$ twice. The filtered extracts were combined and concentrated under rotate reduced pressure to remove ethanol. The concentrated extract was then lyophilized with a Virtis Freeze Dryer (Te Virtis Company, USA) to obtain the final freezedried powder (PNE). PNS was purchased from Yunnan Yunke Pharmaceutical Co. Ltd (China).
2.2. UPLC Analysis. The chemical constitutions of PNS and PNE were quantitatively determined using a Waters ACQUITY-UPLC CLASS system (Waters Corp., USA) coupled with an ACQUITY UPLC BEH phenyl column $(150 \mathrm{~mm} \times 2.1 \mathrm{~mm}, 1.7 \mu \mathrm{m})$ maintained at $45^{\circ} \mathrm{C}$. Elution was performed with a mobile phase of water (A) and acetonitrile (B) under a gradient program: $0-10 \mathrm{~min}, 19 \% \mathrm{~B} ; 10$ $15 \mathrm{~min}, 19-35 \% \mathrm{~B} ; 15-20 \mathrm{~min}, 35-38 \% \mathrm{~B}$. The flow rate was $0.4 \mathrm{~mL} / \mathrm{min}$, and the injection volume was $2 \mu \mathrm{L}$. The analytes were monitored at the UV wavelength of $205 \mathrm{~nm}$. Prior to the next injection, the column was washed with $100 \% \mathrm{~B}$ for $2 \mathrm{~min}$ and then equilibrated with the initial mobile phase for $3 \mathrm{~min}$. Notogisenoside R1, ginsenoside Rb1, ginsenoside $\mathrm{Re}$, ginsenoside $\mathrm{Rg} 1$, and ginsenoside $\mathrm{Rd}$ (the purities of all standards were higher than $98 \%$ by HPLC analysis) were purchased from Chengdu Pufei De Biotech Co., Ltd. (China). Acetonitrile was purchased from RCI Labscan Limited (Thailand) of HPLC grade. Milli-Q water was prepared using a Milli-Q system (Millipore, USA).

2.3. Animals and Drug Treatments. The use of mice and research protocol was approved by the Animal Research Ethics Committee, University of Macau, and conformed to the National Institutes of Health guidelines for the Care of Use of Laboratory Animals. Male C57BL/6J mice were purchased from the Faculty of Healthy Science Animal Centre of the University of Macau and housed in a temperaturecontrolled room $\left(22-24^{\circ} \mathrm{C}\right)$ with a 12-hr light/dark cycle. The mice at the age of 6 weeks were fed with a high-fat diet ( $45 \% \mathrm{kcal} \% \mathrm{fat}$ ) for 12 weeks to establish a type 2 diabetic model followed by oral administration of vehicle (water), PNS, or PNE at $20 \mathrm{mg} / \mathrm{kg}$ body weight daily for another 4 weeks. Mice fed with a normal chow diet served as control.

2.4. Blood Pressure Measurement. Systolic (SBP) and diastolic (DBP) blood pressure were measured by the CODA noninvasive blood pressure system (a tail-cuff method, Kent Scientific Corporation, USA) in conscious mice.

2.5. Blood Glucose Measurement. After 6-h fasting, blood was drawn from the mouse tail to determine fasting blood glucose (FBG) level using a commercial glucometer, and mice with FBG $>11 \mathrm{mM}$ were considered as diabetic. For oral glucose tolerance test (OGTT), mice after 6-h fasting were loaded with glucose solution $(1.2 \mathrm{~g} / \mathrm{kg}$ body weight) by oral gavage and blood glucose levels were measured at $0,15,30,60$, and $120 \mathrm{~min}$. For the insulin tolerance test (ITT), mice after 2-h fasting were injected with insulin $(0.5 \mathrm{U} / \mathrm{kg}$ body weight) intraperitoneal, and the blood glucose levels were detected at the same time intervals as OGTT.

2.6. Ex Vivo Culture of Mouse Aortas. After mice were sacrificed, both thoracic aortas and carotid arteries were rapidly removed and carefully dissected free from adjacent connective tissues in sterile phosphate-buffered saline (PBS). Arteries were cut into segments $(\sim 2 \mathrm{~mm}$ in length) and were incubated in a Dulbecco's Modified Eagle's Media (DMEM, Gibco, USA) supplemented with $10 \%$ fetal bovine serum (FBS, Gibco), plus $100 \mathrm{IU} / \mathrm{mL}$ penicillin and $100 \mu \mathrm{g} / \mathrm{mL}$ streptomycin. Arterial segments were incubated with normal 


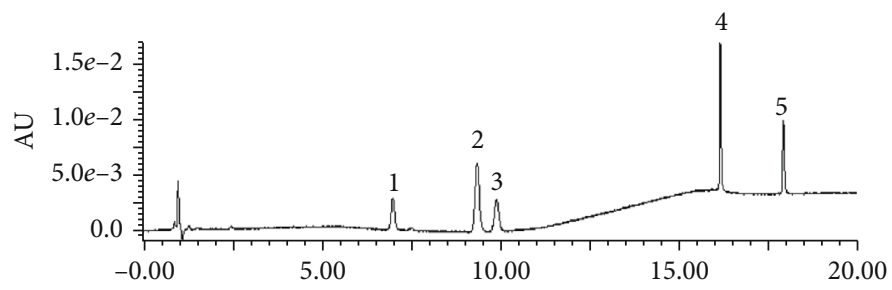

(a)

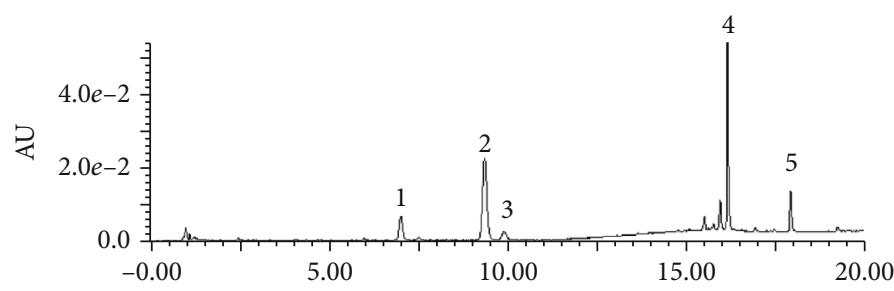

(b)

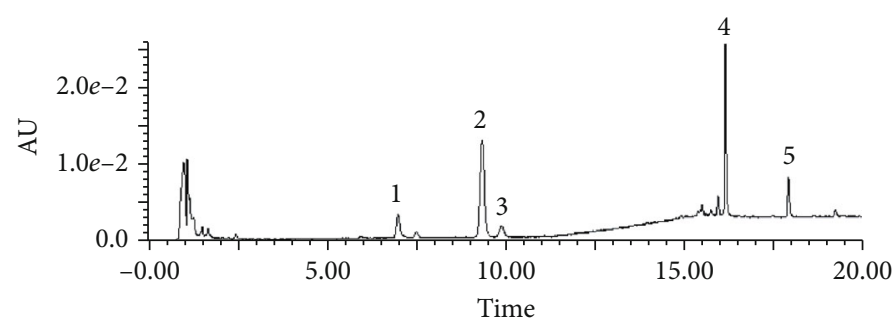

(c)
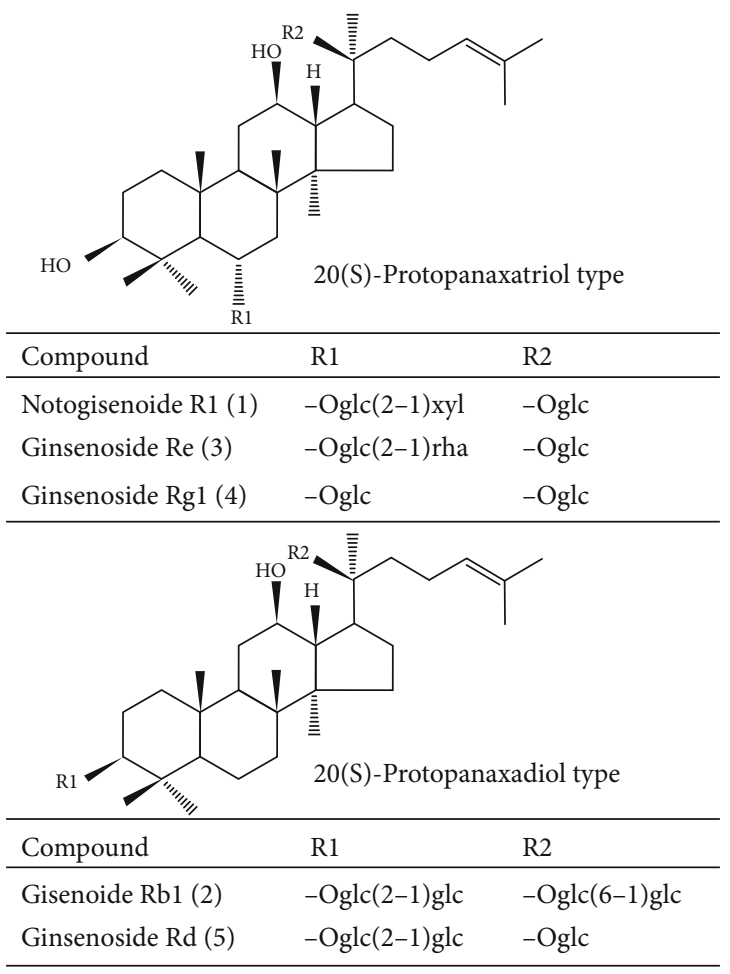

(d)

FIgURe 1: Quantitative analysis of Panax notoginseng ethanolic extract (PNE) and total saponins (PNS). Chromatograms of (a) mixed standards, (b) PNS, and (c) PNE by UPLC analysis. 1: Notogisenoside R1; 2: Ginsenoside Rb1; 3: Ginsenoside Re; 4: Ginsenoside Rg1; 5: Ginsenoside Rd. (d) Chemical structures of notoginsenoside R1, ginsenosides Rg1, Re, Rb1, and Rd. Glc, $\beta$-D-glucose; Rha, $\alpha$-Lrhamnose; Xyl, $\beta$-D-xylose.

medium with mannitol added as osmotic control, high glucose $(30 \mathrm{mM})$ medium or high glucose medium plus individual drugs including different concentrations of PNE or PNS and Compound C ( $5 \mu \mathrm{M}, \mathrm{AMPK}$ inhibitor, Sigma-Aldrich) in incubator at $37^{\circ} \mathrm{C}$ for $48 \mathrm{~h}$. The ring segments were then collected for functional studies in wire myograph, Western blotting, and fluorescence imaging.

2.7. Isometric Force Measurement in Wire Myograph. Aortic segments $(\sim 2 \mathrm{~mm})$ were suspended in a Multi Myograph System (Danish Myo Technology, Denmark) to determine the changes in isometric tension. Mouse aortas were stretched to an optimal baseline tension of $3 \mathrm{mN}$ with equilibration for $60 \mathrm{~min}$ before they were contracted by $60 \mathrm{mM}$ $\mathrm{KCl}$. Endothelium-dependent relaxations (EDRs) were studied in phenylephrine (Phe, $3 \mu \mathrm{M}$, Sigma-Aldrich) precontracted endothelium-intact rings in response to acetylcholine (ACh, $3 \mathrm{nM}-10 \mu \mathrm{M}$, Sigma-Aldrich). Endothelium-independent relaxations were measured in response to sodium nitroprusside (SNP, $1 \mathrm{nM}-10 \mu \mathrm{M}$, Sigma-Aldrich). Each experiment was performed on rings prepared from different mice.

2.8. Western Blot. Aortas snap frozen in liquid nitrogen were subsequently homogenized in ice-cold RIPA lysis buffer. The homogenates were incubated on ice for $30 \mathrm{~min}$ and then centrifuged for $20 \mathrm{~min}$ at $20,000 \mathrm{~g}$. The supernatant was col-
TABLE 1: Contents $(\%, g / g)$ of five main saponins in PNS and PNE.

\begin{tabular}{lcc}
\hline Compound & PNS $(\%, g / g)$ & PNE $(\%, g / g)$ \\
\hline Notogisenoside R1 & $11.20 \pm 0.32$ & $5.45 \pm 0.25$ \\
Ginsenoside Rb1 & $33.51 \pm 0.63$ & $18.96 \pm 0.52$ \\
Ginsenoside Re & $4.16 \pm 0.01$ & $2.18 \pm 0.06$ \\
Ginsenoside Rg1 & $40.57 \pm 0.69$ & $17.71 \pm 0.58$ \\
Ginsenoside Rd & $8.13 \pm 0.14$ & $4.17 \pm 0.16$ \\
Total & 97.57 & 48.47 \\
\hline
\end{tabular}

lected and protein concentration was measured using BCA assay. Protein sample $(15 \mu \mathrm{g})$ was electrophoresed through 7.5\%-12.5\% (SDS-PAGE) and transferred to the PVDF membrane (Millipore) using wet transfer (BIO-RAD, USA). Nonspecific binding sites were blocked by $5 \%$ nonfat milk or $1 \%$ BSA in $0.05 \%$ Tween-20 PBS, and the membranes were then probed with the primary antibodies against target proteins at $4^{\circ} \mathrm{C}$ overnight; followed by incubation with corresponding HRP-conjugated secondary antibodies. The membranes were visualized with an American ECLTM Advanced Western Blotting Detection Kit (GE Healthcare Life Sciences, Sweden) and scanned by ChemiDoc MP Imaging System (BIO-RAD). GAPDH was selected as a housekeeping protein for checking equal loading of each sample. 

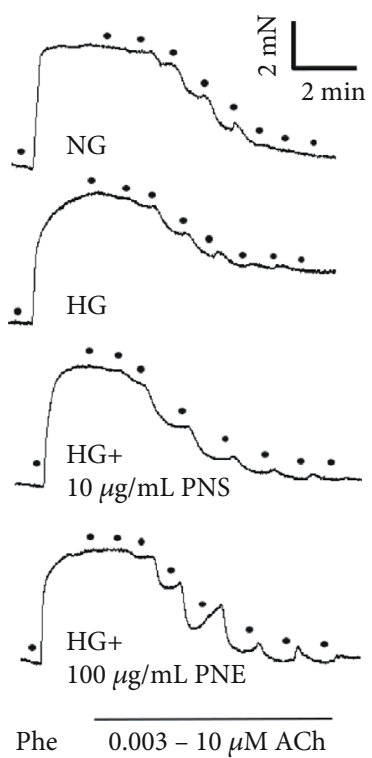

(a)
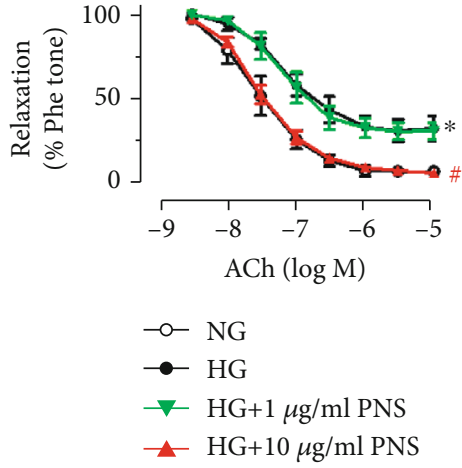

(b)

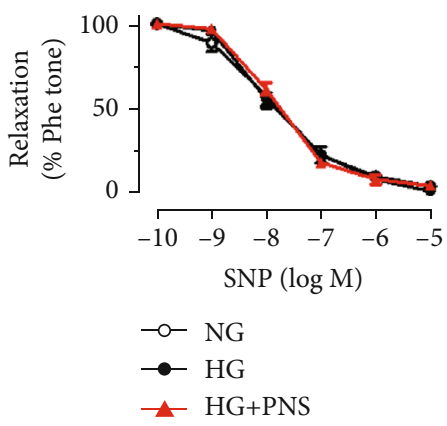

(d)

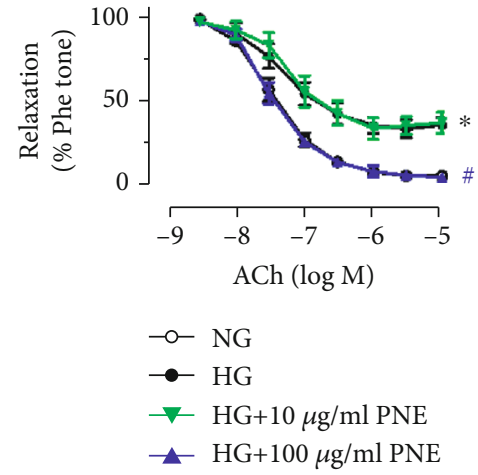

(c)

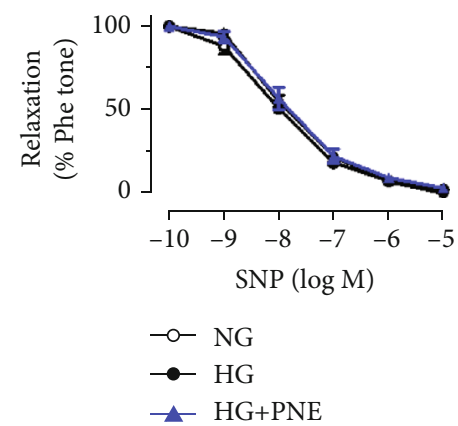

(e)

Figure 2: Panax notoginseng extract protects endothelial function in a hyperglycemic condition. (a) Representative traces and (b, c) summarized data showing the effects of PNS and PNE on acetylcholine- (ACh-) induced EDRs in mouse aortas exposed to high glucose $(\mathrm{HG}, 30 \mathrm{mM}, 48 \mathrm{~h})$. (d, e) Sodium nitroprusside- (SNP-) induced endothelium-independent relaxations in aortas. Data are mean \pm SEM of 4 experiments. ${ }^{*} P<0.05$ vs. normal glucose (NG, $5.55 \mathrm{mM}$ with mannitol); ${ }^{\#} P<0.05$ vs. HG.

\subsection{Culture of Human Umbilical Cord Vein Endothelial Cells} (HUVECs). HUVECs (Lonza) were grown in EGM supplemented with Bulletkit (Lonza) in $75 \mathrm{~cm}^{2}$ flasks and maintained at $37^{\circ} \mathrm{C}$ in a humidified atmosphere of $5 \% \mathrm{CO}_{2}$. Experiments were performed on cells at passage 4-8 to treat with high glucose, PNE, PNS, or Compound C when $80-90 \%$ confluency was achieved.

2.10. Detection of ROS by Dihydroethidium (DHE) Staining. Segments of or aortas (chronic treatment) and carotid arteries (ex vivo treatment) were frozen in OCT compound (Tissue-Tek) and sliced into sections $(10 \mu \mathrm{m})$ using a Leica CM 1000 cryostat. The arterial sections and treated HUVECs were incubated in DHE- (5 $\mu \mathrm{M}$; Invitrogen) containing normal physiological saline solution (NPSS) at $37^{\circ} \mathrm{C}$ for $15 \mathrm{~min}$. NPSS contained (mM): $140 \mathrm{NaCl}, 5 \mathrm{KCl}, 1 \mathrm{CaCl}_{2}, 1 \mathrm{MgCl}_{2}$, 10 glucose, and 5 HEPES (pH 7.4). Fluorescence images were obtained with the Leica TCS SP8 Confocal Laser Scanning Microscope System (Leica Microsystems, Germany) by measuring the fluorescence intensity at excitation $515 \mathrm{~nm}$ and emission $585 \mathrm{~nm}$.

2.11. Statistical Analysis. Data were expressed as mean \pm SEM of $n$-independent experiments. Comparisons were analyzed using one-way ANOVA and $t$-test in the GraphPad Prism software (GraphPad Software, USA). $P<0.05$ was regarded to be a statistically significant difference.

\section{Results}

3.1. Quantitative Analysis of Ethanolic Extract (PNE) and Total Saponin of Panax Notoginseng (PNS). The contents of notogisenoside R1, ginsenoside Rb1, ginsenoside Re, ginsenoside Rg1, and ginsenoside Rd in PNS and PNE were determined using UPLC analysis. The chromatograms of mixed standards, PNS $(0.5 \mathrm{mg} / \mathrm{mL})$ and PNE $(0.5 \mathrm{mg} / \mathrm{mL})$, were illustrated in Figure 1. All compounds were chromatographically separated with the developed analytical method. The contents of five saponins in PNS and PNE were shown in Table 1.

3.2. PNS and PNE Improve Vascular Functions in Hyperglycemic Condition. Aortic rings from C57BL/6J mice were cultured with high glucose $(30 \mathrm{mM}, 48 \mathrm{~h})$ to mimic the hyperglycemic condition in diabetes. High glucoseinduced impairment of EDRs in response to ACh was improved by PNS and PNE (Figures 2(a)-2(c)). PNS exhibited a more potent vaso-protective effect than PNE ex vivo. PNS had no effect at $1 \mu \mathrm{g} / \mathrm{mL}$ but was effective at $10 \mu \mathrm{g} / \mathrm{mL}$ to restore EDRs comparable to the control (5.55 mM glucose in DMEM with the addition of mannitol as osmotic control). By contrast, PNE ameliorated high glucose-induced endothelial dysfunction at a higher concentration of $100 \mu \mathrm{g} / \mathrm{mL}$. Endothelium-independent relaxations to SNP (Figures 2(d) and $2(\mathrm{e})$ ) were not altered among groups, indicating the response of vascular smooth muscle to NO was normal. 


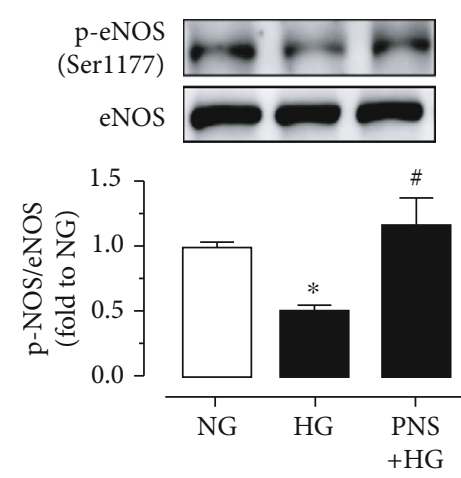

(a)

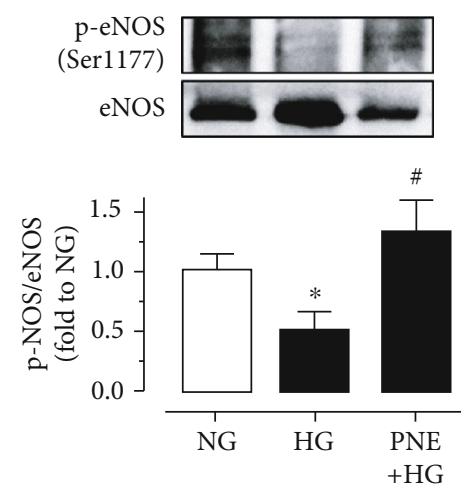

(d)

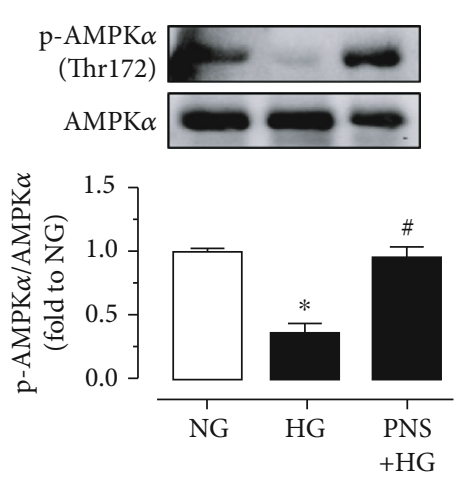

(b)

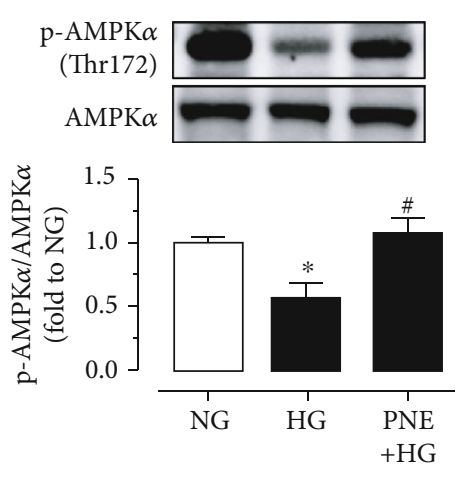

(e)
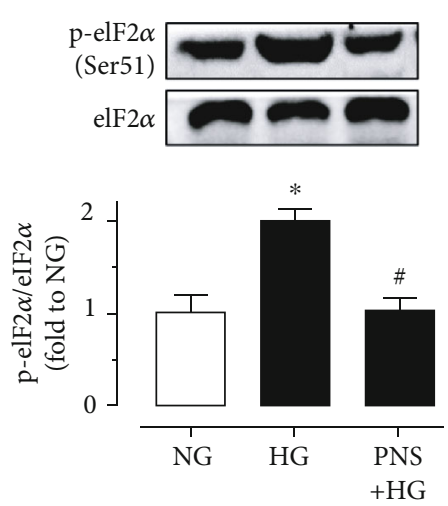

(c)

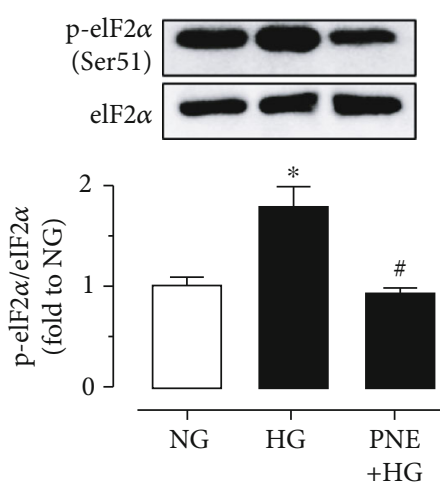

(f)

FIgURE 3: PNS and PNE stimulate AMPK $\alpha /$ eNOS and reduce ER stress. Western blotting showing (a) the phosphorylation of eNOS at Ser1177 (p-eNOS; $140 \mathrm{kDa}$ ), (b) phosphorylation of AMPK $\alpha$ at Thr172 (p-AMPK $\alpha ; 62 \mathrm{kDa}$ ), and (c) phosphorylation of eIF2 $\alpha$ at Ser52 (p-eIF $2 \alpha ; 36 \mathrm{kDa})$ compared to their corresponding total protein in mouse aortas treated with high glucose (HG, $30 \mathrm{mM})$ and PNS $(10 \mu \mathrm{g} / \mathrm{mL})$ for $48 \mathrm{~h}$. (d-f) Protein expressions in mouse aortas treated with PNE $(100 \mu \mathrm{g} / \mathrm{mL})$. Data are mean \pm SEM of 4 experiments. ${ }^{*}$ $P<0.05$ vs. normal glucose (NG, $5.5 \mathrm{mM}$ with mannitol); ${ }^{\#} P<0.05$ vs. HG.

The lowest effective concentrations of PNS and PNE observed in the functional studies were chosen for further experiments to study the underlying mechanisms: $10 \mu \mathrm{g} / \mathrm{mL}$ and $100 \mu \mathrm{g} / \mathrm{mL}$, respectively.

3.3. PNS and PNE Enhance AMPK/eNOS Pathway and Attenuate ER Stress. Upon treatment with high glucose for $48 \mathrm{~h}$, the phosphorylation of eNOS at Ser1177 and phosphorylation of AMPK $\alpha$ at Thr172 was suppressed, while phosphorylated eukaryotic initiation factor $2 \alpha(\mathrm{eIF} 2 \alpha)$ at Ser52 was elevated in mouse aortas (Figure 3). Such changes were reversed by co-treatment with PNS $(10 \mu \mathrm{g} / \mathrm{mL})$ (Figures 3(a)-3(c)) and PNE $(100 \mu \mathrm{g} / \mathrm{mL})$ (Figures 3(d)-3(f)). In addition, the vasoprotective effect of PNS and PNE against high glucoseinduced impairment of EDRs was abolished by AMPK inhibitor Compound C (5 $\mathrm{M})$ (Figures 4(a) and 4(b)). Ex vivo 24-h exposure of mouse aortas to the ER stress inducer tunicamycin $(2 \mu \mathrm{g} / \mathrm{mL})$ reduced EDRs, which were restored by coincubation with PNS $(10 \mu \mathrm{g} / \mathrm{mL}$ ) (Figure $4(\mathrm{c}))$ and PNE $(100 \mu \mathrm{g} / \mathrm{mL})$ (Figure $4(\mathrm{~d}))$ without affecting SNPinduced endothelium-independent relaxations (Figure 4(e)). Similarly, the improvements in EDRs were inhibited by Compound C.
3.4. PNS and PNE Suppress Oxidative Stress through an AMPK-Dependent Mechanism. Both PNS $(10 \mu \mathrm{g} / \mathrm{mL})$ and PNE $(100 \mu \mathrm{g} / \mathrm{mL})$ effectively inhibited high glucose$(30 \mathrm{mM}, 4 \mathrm{~h})$ induced ROS elevation in mouse carotid arteries (Figure 5(a)) and in HUVECs (Figures 5(b) and 5(c)). PNS was more potent to reduce oxidative stress at $10 \mu \mathrm{g} / \mathrm{mL}$, while PNE was only effective at $100 \mu \mathrm{g} / \mathrm{mL}$. The antioxidative effects of both PNS and PNE were reversed by Compound $\mathrm{C}(5 \mu \mathrm{M})$, indicating that AMPK activation contributes to the antioxidative activity of PNS and PNE.

3.5. Chronic Treatment with PNS and PNE Ameliorates Endothelial Dysfunction and Oxidative Stress in DietInduced Obese Mice. To examine the vascular benefit of PNS and PNE treatments in vivo, diet-induced obese (DIO) mice were orally administered with PNS and PNE at $20 \mathrm{mg} / \mathrm{kg} /$ day for 4 weeks. Feeding with a high-fat diet for 16 weeks markedly increased body weight as compared to the control mice feeding with normal chow whereas PNS and PNE treatments exerted no effect on body weight (Figure 6(a)). In contrast to the in vitro data, chronic PNE treatment showed more effective protection of endothelial function and glycemic control than PNS. Four-week PNE 


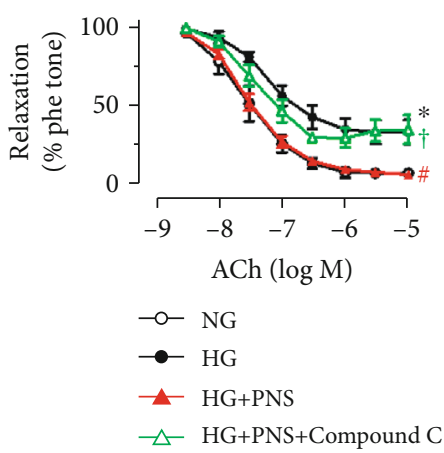

(a)

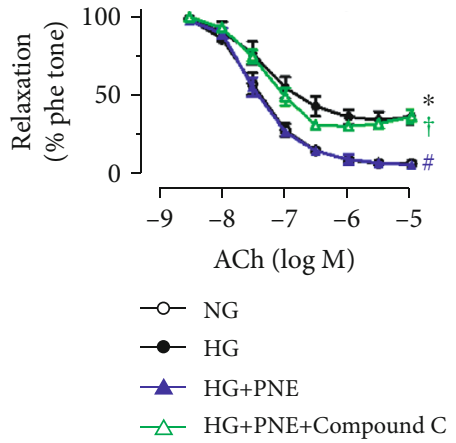

(b)

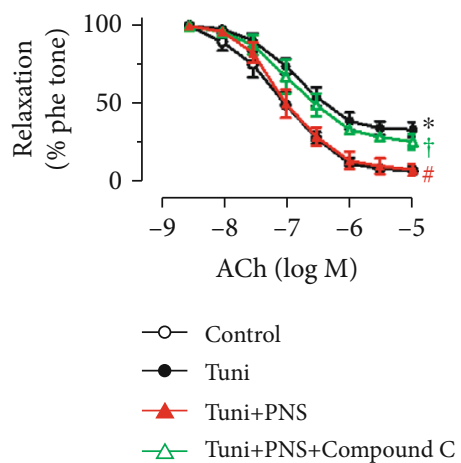

(c)

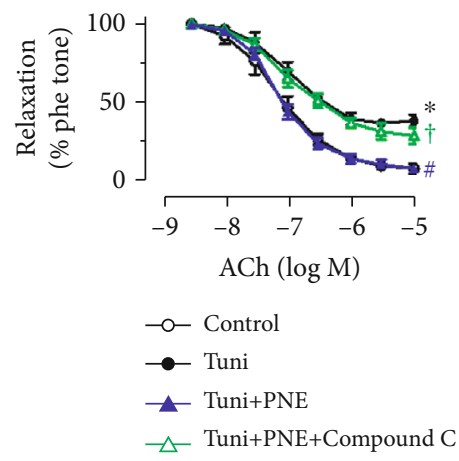

(d)

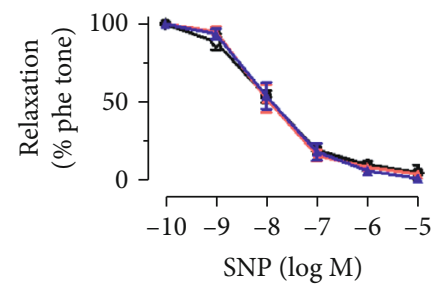

$-\circ$ Control

$\rightarrow$ Tuni

- Tuni+PNE

$\rightarrow-$ Tuni+PNS

(e)

FIGURE 4: AMPK and ER stress modulates the beneficial effect of PNS and PNE on endothelial function. (a, b) Effect of AMPK inhibitor Compound $\mathrm{C}(5 \mu \mathrm{M})$ on ACh-induced EDRs in mouse aortas treated with high glucose (HG, $30 \mathrm{mM}), \mathrm{PNS}(10 \mu \mathrm{g} / \mathrm{mL})$ and PNE $(100 \mu \mathrm{g} / \mathrm{mL})$ for $48 \mathrm{~h}$. Effect of pretreatment with ER stress inducer tunicamycin (Tuni, $2 \mu \mathrm{g} / \mathrm{mL}, 24 \mathrm{~h}$ ) and cotreatment with PNS $(10 \mu \mathrm{g} / \mathrm{mL})$ or PNE $(100 \mu \mathrm{g} / \mathrm{mL})$ on $(C$ and D) ACh-induced and (e) SNP-induced relaxations in mouse aortas. Data are mean \pm SEM of 4 experiments. ${ }^{*} P<0.05$ vs. normal glucose (NG, $5.5 \mathrm{mM}$ with mannitol) or control; ${ }^{\#} P<0.05$ vs. HG or Tuni; ${ }^{\dagger} P<0.05$ vs. $\mathrm{HG}+\mathrm{PNS}$, $\mathrm{HG}+\mathrm{PNE}$, Tuni+PNS, or Tuni+PNE.

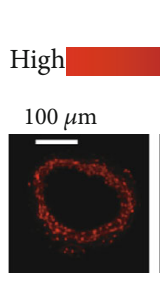

NG

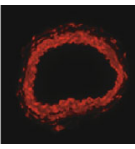

HG
ROS

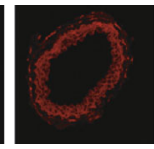

PNE $+\mathrm{HG}$$$
\text { ษัّ }
$$

Carotid arteries

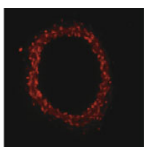

PNS $+\mathrm{HG}$

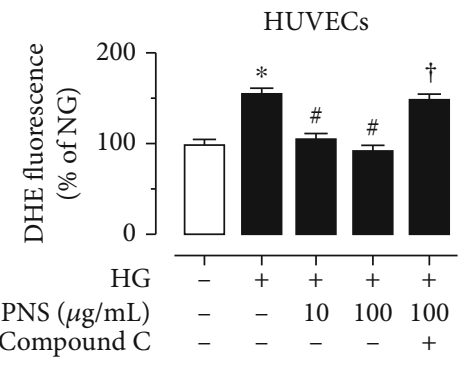

(b)

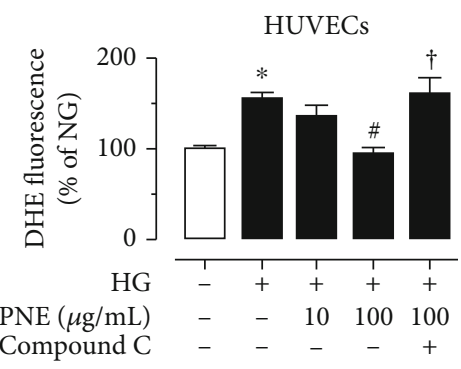

(c)

(a)

FIGURE 5: PNS and PNE attenuate oxidative stress. (a) Representative images and summarized graph showing DHE intensity (oxidative stress indicator) in mouse carotid arteries treated with high glucose $(30 \mathrm{mM}, 4 \mathrm{~h}), \mathrm{PNS}(10 \mu \mathrm{g} / \mathrm{mL})$, and $\mathrm{PNE}(100 \mu \mathrm{g} / \mathrm{mL})$. (b, c) Inhibitive effects of PNS and PNE on high glucose-induced oxidative stress in HUVECs. Data are mean \pm SEM of 4 experiments. ${ }^{*} P<$ 0.05 vs. normal glucose (NG, $5.5 \mathrm{mM}$ with mannitol); ${ }^{\#} P<0.05$ vs. HG. 


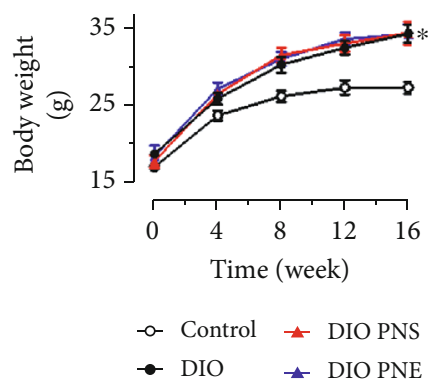

(a)

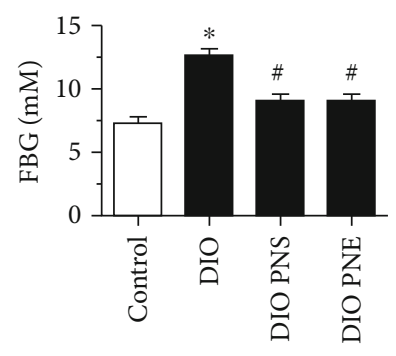

(d)

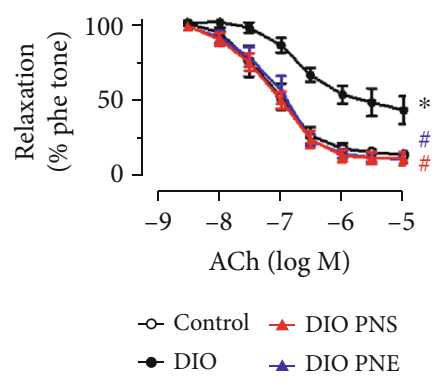

(g)

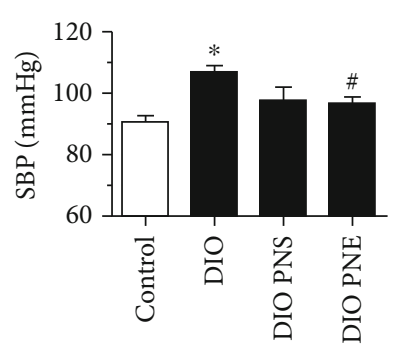

(b)

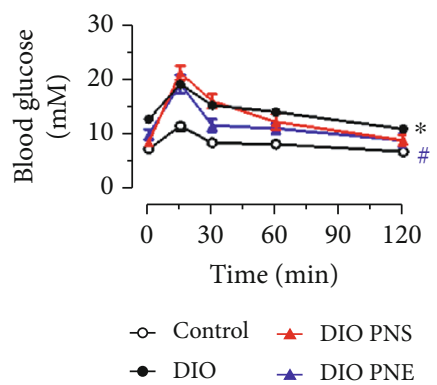

(e)

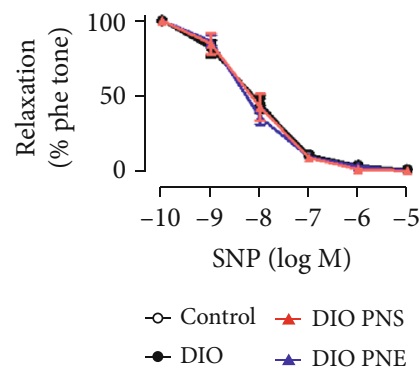

(h)

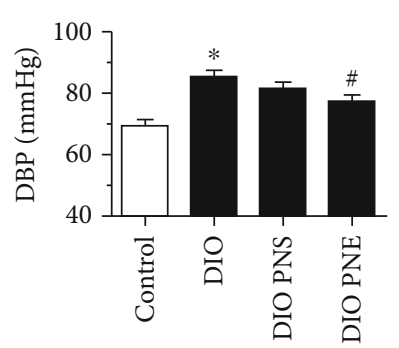

(c)

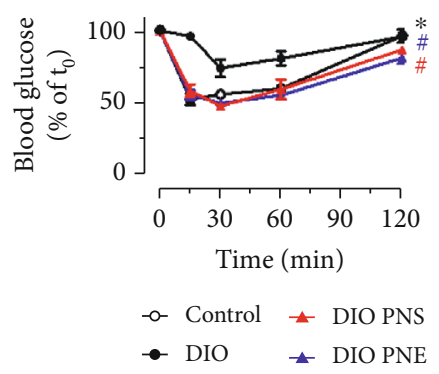

(f)

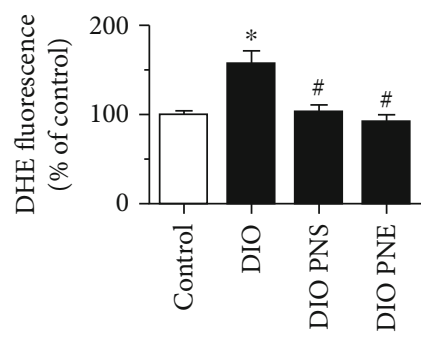

(i)

FIGURE 6: Vaso-protective effect of PNS and PNE in diet-induced obese (DIO) mice. (a) Changes in body weight in mice fed with high-fat or normal chow diet for 16 weeks and administered with vehicle (water), PNS, or PNE at $20 \mathrm{mg} / \mathrm{kg}$ body weight daily by oral gavage at the last 4 weeks. (b, c) Changes in systolic (SBP) and diastolic (DBP) blood pressure measured by tail-cuff method. (d) Changes in fast blood glucose (FBG) measured after fasting the mice for $6 \mathrm{~h}$. (e) Oral glucose tolerance test (OGTT) upon 6-h fasting. (f) Insulin tolerance test (ITT) upon 2-h fasting. (g) Effect of oral administration of PNS or PNE $(20 \mathrm{mg} / \mathrm{kg} / \mathrm{day}$, 4 weeks) on ACh-induced relaxations in aortas from DIO mice. (h) SNP-induced endothelium-independent relaxations in mouse aortas. (i) Changes in DHE intensity (oxidative stress indicator) in mouse aortas. Data are mean \pm SEM of 5 experiments. ${ }^{*} P<0.05$ vs. control; ${ }^{\#} P<0.05$ vs. DIO.

treatment normalized the elevated systolic and diastolic blood pressures in DIO mice, while PNS exhibited a small but insignificant effect (Figures 6(b) and 6(c)). Fasting glucose levels of DIO mice were higher than those of control and were greater than $11 \mathrm{mM}$, indicating the success of establishing a diabetic model in mice (Figure 6(d)). Oral glucose (Figure 6(e)) and insulin tolerance tests (Figure 6(f)) revealed impaired glucose sensitivity and insulin irresponsiveness in DIO mice comparing to lean nondiabetic control mice. Both PNS and PNE treatments normalized the elevated fasting glucose level and improved insulin sensitivity; nevertheless, the reduced glucose tolerance was moderately reversed by $\mathrm{PNE}$ treatment but was insensitive to PNS treatment. ACh-induced EDRs were decreased in DIO mice and chronic PNS and PNE treatments restored the impaired EDRs (Figure 6(g)) without affecting SNPinduced endothelium-independent relaxations in aortas (Figure 6(h)). The elevated ROS level was also reversed by PNS and PNE treatments in aortas from DIO mice (Figure 6(i)).

\subsection{PNS and PNE Activate AMPK/eNOS and Alleviate ER} Stress in Aortas of DIO Mice. In aortas from DIO mice, eNOS phosphorylation at Ser1177 and AMPK $\alpha$ phosphorylation at Thr172 were downregulated (Figures $7(\mathrm{a})$ and 7(b)), while ER stress markers including cleaved activating transcription factor 6 (ATF6) and eIF2 $\alpha$ phosphorylation at Ser52 were upregulated compared with lean control (Figures $7(\mathrm{c})$ and $7(\mathrm{~d})$ ). PNS and PNE treatments reversed these changes on AMPK/eNOS and ER stress in DIO mice.

\section{Discussion}

The present study used multiple approaches to elucidate and compare the effects of PNS and PNE on endothelial dysfunction in diet-induced obese mice. Treatment with either PNS 


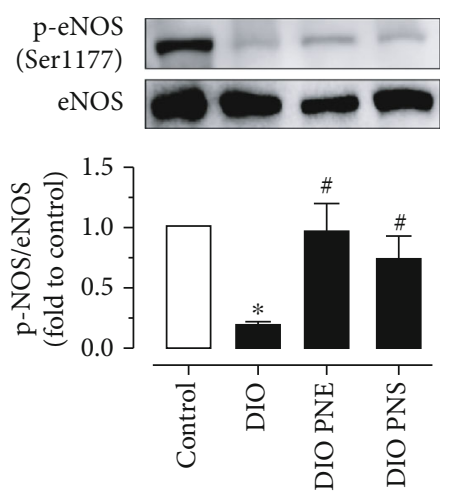

(a)

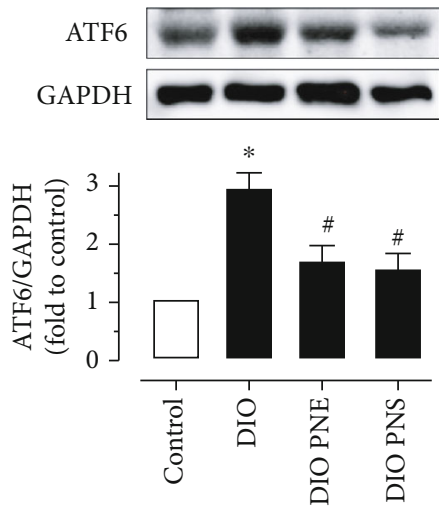

(c)
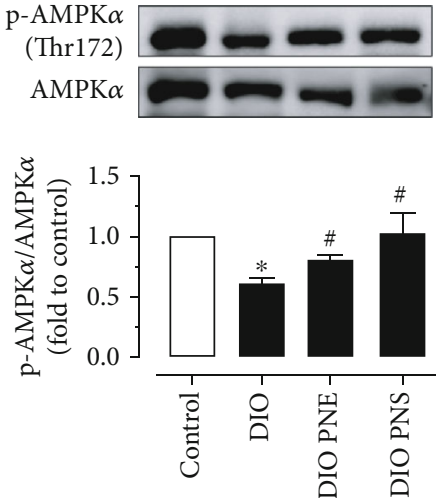

(b)
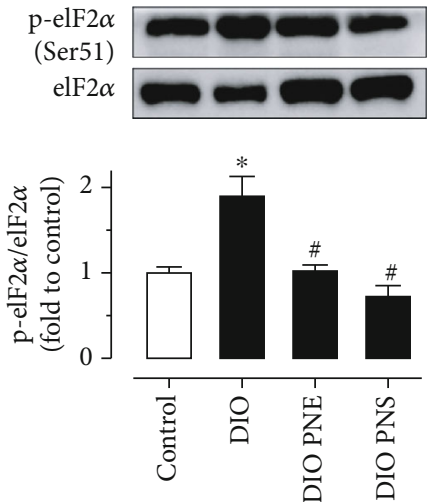

(d)

FIgURE 7: Chronic PNS and PNE treatments activate AMPK/eNOS pathway and reduce ER stress. Western blot data showing (a) the phosphorylation at Ser1177 and total eNOS (140 kDa), (b) phosphorylation at Thr172 and total AMPK $\alpha$ (62 kDa), (c) cleaved ATF6 $(50 \mathrm{kDa})$ compared to GAPDH, and (d) phosphorylation at Ser52 and total eIF2 $\alpha(36 \mathrm{kDa})$ in aortas from four groups of mice. Data are mean \pm SEM of 5 experiments. ${ }^{*} P<0.05$ vs. control; ${ }^{\#} P<0.05$ vs. DIO.

or PNE rescued the impaired endothelial function in a hyperglycemic condition and in diabetic obese mice, accompanied by enhancement of vascular AMPK/eNOS activities with inhibition of ER stress and oxidative stress. PNS was found to be more potent ex vivo and in vitro but PNE was more effective in vivo.

With the advances of scientific development and emergence of new extraction or identification methods, an increasing number of novel compounds are being discovered from $P$. notoginseng. The chemical composition varies in different parts of $P$. notoginseng and is affected by the processing method. Until now, more than 200 phytochemicals have been isolated and identified from various parts of P. notoginseng, including saponins, polysaccharides, dencichine (a nonprotein amino acid), flavonoids, fatty acids, and volatile oil $[1,26]$. According to the data of UPLC analysis, PNS used in the present study mainly contains five saponins including notogisenoside R1 and ginsenosides Rb1, Re, Rg1, and Rd, while the five saponins account for around half of the contents in PNE. Another half of PNE probably consists of other chemical components such as polysaccharides, dencichine, flavonoids, fatty acids, and volatile oil, which needs to be identified by further investigation.

AMPK activation stimulates eNOS and increases NO bioavailability [23]. Moreover, AMPK has been widely con- firmed to decrease ROS production in diabetes-related diseases [27-29]. Previous studies showed that AMPK activation suppresses ER stress and control of ER stress is pivotal for vascular function $[24,25,30]$. PNS was described to promote angiogenesis in HUVECs [31] and protect against acute myocardial infarction in mice [8] through AMPK activation. Our observations are in line with these previous studies, reporting that ex vivo high glucose exposure impaired EDRs and stimulated ROS production, which were reversed by cotreatment of PNS and PNE. Importantly, these protective effects of PNS and PNE were absent when AMPK inhibitor Compound $\mathrm{C}$ was added. PNS and PNE suppressed the phosphorylation of $\operatorname{eIF} 2 \alpha$, one of the ER stress markers triggered by high glucose exposure. Likewise, induction of ER stress by tunicamycin impaired EDRs in conduit aortas which were rescued by PNS and PNE, and such improvement was abolished in the presence of Compound $\mathrm{C}$. The present results show that both PNS and PNE effectively improved high glucose-induced endothelial dysfunction with alleviation of ER stress and oxidative stress through the AMPK/eNOS pathway. Besides, PNS was more potent to exert vaso-protective effects at $10 \mu \mathrm{g} / \mathrm{mL}$ but PNE showed no improvement at the same concentration.

Excessive nutrient such as glucose in diabetes is one of the common risk factors causing vascular complications 
[32]. Chronic treatment with PNS and PNE did not affect body weight but improved glucose metabolism, which might partially contribute to the in vivo beneficial effects to improve EDRs and normalize blood pressure in diabetic and obese mice. The in vivo study showed contradictory findings with the ex vivo and in vitro studies. Treatment of DIO mice with the same amount of PNS and PNE $(20 \mathrm{mg} / \mathrm{kg} /$ day for 4 weeks) potently improved EDRs and reduced ROS levels to the similar degree. However, PNE but not PNS significantly reduced systolic and diastolic blood pressure. This may be explained by the difference of effects on glucose metabolism for these two extracts: PNE moderately improved glucose sensitivity but PNS did not. The ex vivo experiments showed treatment of mouse aortas with tunicamycin impaired EDRs, which were improved by PNS and PNE, where ambient glucose was constant. Therefore, these results strongly suggest that PNS and PNE have direct vaso-protective effects through alleviation of ER stress and oxidative stress even without affecting glucose metabolism. Western blot data showed that chronic treatment with PNS and PNE resulted in increased phosphorylation of eNOS and AMPK as well as decreased levels of both ER stress and oxidative stress, indicating that increased AMPK/eNOS activity and suppressed ER stress are likely to mediate the vaso-protective effects of PNS and PNE.

PNS is commonly considered as the major pharmacological active ingredients of $P$. notoginseng. However, our present study demonstrates that the whole ethanolic extract containing both saponins and other chemical compounds exhibited better beneficial effects in improving glucose metabolism, reversing hypertensive condition, and protecting endothelial function. Vascular function is regulated by multiple factors including glucose metabolism [33, 34]. The additional benefits of PNE may be attributed to the presence of other active ingredients apart from saponins, or saponins may interact with other ingredients to have synergistic effects in improving vascular function in diabetes. This possibility remains to be further explored.

\section{Conclusions}

The present results show that PNS and PNE protected against diabetes-associated endothelial dysfunction through activating the AMPK/eNOS pathway, accompanied by alleviation of ER stress and oxidative stress. The current novel findings support the therapeutic potential of $P$. notoginseng in combating vascular and metabolic diseases.

\section{Data Availability}

The data used to support the content of this manuscript are available upon request to the corresponding author.

\section{Conflicts of Interest}

The authors declare no conflict of interest.

\section{Authors' Contributions}

Xutao Zhang and Chunxiu Zhou contributed equally to this work. Xutao Zhang and Chunxiu Zhou are co-first authors.

\section{Acknowledgments}

The authors thank the Animal Research Core in the Faculty of Health Sciences, University of Macau. This work was supported by the Research Committee of the University of Macau (grant numbers SRG2019-00154-ICMS and MYRG201900157-ICMS), Hong Kong RGC-Senior Research Fellow Scheme (grant number SRFS2021-4S04), the Major basic and applied basic research projects of Guangdong Province of China (grant number 2019B030302005), and the Science and Technology Development Fund, Macau SAR [grant numbers 0013/2019/AFJ and SKL-QRCM(UM)-2020-2022].

\section{References}

[1] T. Wang, R. Guo, G. Zhou et al., "Traditional uses, botany, phytochemistry, pharmacology and toxicology of Panax notoginseng (Burk.) F.H. Chen: a review," Journal of Ethnopharmacology, vol. 188, pp. 234-258, 2016.

[2] X. Zuo, Q. Li, F. Ya et al., "Ginsenosides Rb2 and Rd2 isolated fromPanax notoginsengflowers attenuate platelet function through P2Y12-mediated cAMP/PKA and PI3K/Akt/Erk1/2 signaling," Food \& Function, vol. 12, no. 13, pp. 5793-5805, 2021.

[3] W. Wang, L. Yang, L. Song et al., "Combination of Panax notoginseng saponins and aspirin potentiates platelet inhibition with alleviated gastric injury via modulating arachidonic acid metabolism," Biomedicine \& Pharmacotherapy, vol. 134, article 111165, 2021.

[4] Z.-y. Xu, Y. Xu, X.-f. Xie et al., “Anti-platelet aggregation of Panax notoginseng triol saponins by regulating GP1BA for ischemic stroke therapy," Chinese Medicine, vol. 16, no. 1, p. 12, 2021.

[5] X. Zhang, B. Zhang, C. Zhang, G. Sun, and X. Sun, "Effect of Panax notoginseng saponins and major anti-obesity components on weight loss," Frontiers in Pharmacology, vol. 11, 2020.

[6] R. Uzayisenga, P. A. Ayeka, and Y. Wang, "Anti-diabetic potential of Panax notoginseng saponins (PNS): a review," Phytotherapy Research, vol. 28, no. 4, pp. 510-516, 2014.

[7] Y. Xu, N. Wang, H.-Y. Tan, S. Li, C. Zhang, and Y. Feng, "Gutliver axis modulation of Panax notoginseng saponins in nonalcoholic fatty liver disease," Hepatology International, vol. 15, no. 2, pp. 350-365, 2021.

[8] D. Wang, L. Lv, Y. Xu et al., "Cardioprotection of Panax Notoginseng saponins against acute myocardial infarction and heart failure through inducing autophagy," Biomedicine \& Pharmacotherapy, vol. 136, article 111287, 2021.

[9] T. Sun, P. Wang, T. Deng, X. Tao, B. Li, and Y. Xu, "Effect of Panax notoginseng saponins on focal cerebral ischemiareperfusion in rat models: a meta-analysis," Frontiers in Pharmacology, vol. 11, p. 2491, 2021.

[10] B.-r. Yang, S.-j. Hong, S. M.-Y. Lee et al., "Pro-angiogenic activity of notoginsenoside $\mathrm{R} 1$ in human umbilical vein endothelial cells in vitro and in a chemical-induced blood vessel loss model of zebrafish in vivo," Chinese Journal of Integrative Medicine, vol. 22, no. 6, pp. 420-429, 2016. 
[11] Y. Yu, G. Sun, Y. Luo et al., "Cardioprotective effects of Notoginsenoside R1 against ischemia/reperfusion injuries by regulating oxidative stress- and endoplasmic reticulum stressrelated signaling pathways," Scientific Reports, vol. 6, no. 1, 2016.

[12] J. Chen, L. Li, X. Bai et al., "Inhibition of autophagy prevents Panax notoginseng saponins (PNS) protection on cardiac myocytes against endoplasmic reticulum (ER) stress-induced mitochondrial injury, $\mathrm{Ca} 2+$ homeostasis and associated apoptosis," Frontiers in Pharmacology, vol. 12, 2021.

[13] C. D. Ochoa, R. F. Wu, and L. S. Terada, "ROS signaling and ER stress in cardiovascular disease," Molecular Aspects of Medicine, vol. 63, pp. 18-29, 2018.

[14] K. W. Choy, D. Murugan, and M. R. Mustafa, "Natural products targeting ER stress pathway for the treatment of cardiovascular diseases," Pharmacological Research, vol. 132, pp. 119-129, 2018.

[15] D. J. Ho, X. Cao, C. Perez, R. V. Sharma, R. L. Davisson, and A. L. Mark, Diet-induced obesity (DIO) produces endoplasmic reticulum (ER) stress in a key brain cardiovascular control region, Lippincott Williams \& Wilkins, Philadelphia, PA, USA, 2010.

[16] G. S. Hotamisligil, "Endoplasmic reticulum stress and the inflammatory basis of metabolic disease," Cell, vol. 140, no. 6, pp. 900-917, 2010.

[17] Y. Zhou, D. D. Murugan, H. Khan, Y. Huang, and W. S. Cheang, "Roles and therapeutic implications of endoplasmic reticulum stress and oxidative stress in cardiovascular diseases," Antioxidants, vol. 10, no. 8, p. 1167, 2021.

[18] C. X. Santos, A. A. Nabeebaccus, A. M. Shah, L. L. Camargo, S. V. Filho, and L. R. Lopes, "Endoplasmic reticulum stress and Nox-mediated reactive oxygen species signaling in the peripheral vasculature: potential role in hypertension," Antioxidants \& Redox Signaling, vol. 20, no. 1, pp. 121-134, 2014.

[19] X. Xue, J. H. Piao, A. Nakajima et al., "Tumor necrosis factor $\alpha$ $(\mathrm{TNF} \alpha)$ induces the unfolded protein response (UPR) in a reactive oxygen species (ROS)-dependent fashion, and the UPR counteracts ROS accumulation by TNF $\alpha$," The Journal of Biological Chemistry, vol. 280, no. 40, pp. 33917-33925, 2005.

[20] J. N. Peoples, A. Saraf, N. Ghazal, T. T. Pham, and J. Q. Kwong, "Mitochondrial dysfunction and oxidative stress in heart disease," Experimental \& Molecular Medicine, vol. 51, no. 12, pp. 1-13, 2019.

[21] W. S. Cheang, W. T. Wong, L. Zhao et al., "PPAR $\delta$ is required for exercise to attenuate endoplasmic reticulum stress and endothelial dysfunction in diabetic mice," Diabetes, vol. 66, no. 2, pp. 519-528, 2017.

[22] W. S. Cheang, X. Y. Tian, W. T. Wong et al., "Metformin protects endothelial function in diet-induced obese mice by inhibition of endoplasmic reticulum stress through $5^{\prime}$ adenosine monophosphate-activated protein kinase-peroxisome proliferator-activated receptor $\delta$ pathway," Arteriosclerosis, Thrombosis, and Vascular Biology, vol. 34, no. 4, pp. 830836, 2014.

[23] Z. Chen, I. C. Peng, W. Sun et al., "AMP-activated protein kinase functionally phosphorylates endothelial nitric oxide synthase Ser633," Circulation Research, vol. 104, no. 4, pp. 496-505, 2009.

[24] Y. Dong, M. Zhang, S. Wang et al., "Activation of AMPactivated protein kinase inhibits oxidized LDL-triggered endo- plasmic reticulum stress in vivo," Diabetes, vol. 59, no. 6, pp. 1386-1396, 2010.

[25] H. J. Hwang, T. W. Jung, J. W. Kim et al., "Protectin DX prevents $\mathrm{H}_{2} \mathrm{O}_{2}$-mediated oxidative stress in vascular endothelial cells via an AMPK-dependent mechanism," Cellular Signalling, vol. 53, pp. 14-21, 2019.

[26] C. Z. Wang, E. McEntee, S. Wicks, J. A. Wu, and C. S. Yuan, "Phytochemical and analytical studies of Panax notoginseng (Burk.) F.H. Chen," Journal of Natural Medicines, vol. 60, no. 2, pp. 97-106, 2006.

[27] X. Li, J. Liu, Q. Lu et al., "AMPK: a therapeutic target of heart failure-not only metabolism regulation," Bioscience Reports, vol. 39, no. 1, 2019.

[28] R. C. Rabinovitch, B. Samborska, B. Faubert et al., "AMPK maintains cellular metabolic homeostasis through regulation of mitochondrial reactive oxygen species," Cell Reports, vol. 21, no. 1, pp. 1-9, 2017.

[29] Y. Ren and H.-M. Shen, "Critical role of AMPK in redox regulation under glucose starvation," Redox Biology, vol. 25, article 101154, 2019.

[30] C. K. Cheng, J. Y. Luo, C. W. Lau et al., "A GLP-1 analog lowers ER stress and enhances protein folding to ameliorate homocysteine-induced endothelial dysfunction," Acta Pharmacologica Sinica, 2021.

[31] D. Wang, Q. Jie, B. Liu et al., "Saponin extract from Panax notoginseng promotesangiogenesis through AMPK- and eNOS-dependent pathways in HUVECs," Molecular Medicine Reports, vol. 16, no. 4, pp. 5211-5218, 2017.

[32] C. M. Sena, A. M. Pereira, and R. Seica, "Endothelial dysfunction - a major mediator of diabetic vascular disease," Biochimica et Biophysica Acta, vol. 1832, no. 12, pp. 2216-2231, 2013.

[33] R. J. Pickering, "Oxidative stress and inflammation in cardiovascular diseases," Antioxidants, vol. 10, no. 2, p. 171, 2021.

[34] X. Du, A. Patel, C. S. Anderson, J. Dong, and C. Ma, "Epidemiology of cardiovascular disease in China and opportunities for improvement: JACC International," Journal of the American College of Cardiology, vol. 73, no. 24, pp. 3135-3147, 2019. 